\title{
From Network Reconstruction to Network Econometrics: Unbiased Estimation of Average Effects $+$
}

\author{
Marzio Di Vece ${ }^{1}$, Tiziano Squartini 1,2 and Diego Garlaschelli 1,3 \\ IMT School for Advanced Studies Lucca, Piazza San Francesco 19, Lucca, Italy \\ 2 Institute for Advanced Study (IAS), University of Amsterdam, Amsterdam, The Netherlands \\ 3 Instituut-Lorentz for Theoretical Physics, Leiden University, Niels Bohrweg 2, Leiden, The Netherlands \\ + Presented at the Entropy 2021: The Scientific Tool of the 21st Century, 5-7 May 2021; Available online: \\ https://sciforum.net/conference/Entropy2021/.
}

Published: 5 May 2021

The International Trade Network (ITN) can be thought of as a set of countries (nodes) that are linked among each other by trade relationships (links). Such a network can be described at the topological level, discerning the partners of each country, and at the weighted level, where our interest shifts to the trade volume among partners.

In Economics, the focus has traditionally been on the estimation of trade volumes and marginal effects, at times forgetting the importance of the network structure. A correct estimation of such a structure increases the precision of the estimation of the parameters and hence of trade volumes. Even if the Econometric models can reproduce the expected number of zeros, they cannot reproduce ITN topological statistics.

An alternative way of studying the ITN structure has been advanced by the Network Science community and consists in a constrained maximization of the Graph Entropy. A recent development is the so-called Enhanced Gravity Model (EGM) where the Lagrange Multipliers are expressed in terms of the rescaled GDPs. The EGM has been proven to accurately predict both topological and weighted statistics. Here we rephrase the EGM into an Econometric model at different degrees of topological detail. Such a model can be used for unbiased estimation of covariate effects on trade volumes taking into account the possible bias given by the underlying topological structure.

Our results confirm that Network Econometric Models replicate the principal network statistics and are associated with the smallest AIC and BIC scores with respect to Econometric models commonly used in International Trade such asPoisson, Negative Binomial and Zero-Inflated.

Our contribution sheds light on how International Trade Econometrics can be updated and completed with Network Regression Models derived from a constrained Maximum Entropy principle. 